\title{
Singular Integrals and the Principal Series, II
}

\author{
A. W. Knapp* and E. M. Stein $\dagger$
}

CORNELL UNIVERSITY, ITHACA, NEW YORK, AND PRINCETON UNIVERSITY, PRINCETON, NEW JERSEY

Communicated by D. C. Spencer, February 16, 1970

Abstract. A further study is made of the intertwining operators for the representations of the simple Lie groups of real rank one. We extend and apply this to the higher rank case, obtaining various results dealing with reducibility of principal series and existence of complementary series.

In two earlier notes, ${ }^{1,2}$ we dealt with theorems concerning existence of representations of the complementary series and irreducibility of the principal series of any connected semisimple Lie group of real-rank one. In the present note we give further theorems about the rank one case, and we show how this gives information about semisimple groups of arbitrary rank.

Our results are as follows. (a) For the general case we study the intertwining integrals, show how to normalize them, and prove the relations among them. (b) For a complex semisimple group, whenever the formal condition of symmetry obtains, there is a complementary series. This is not so for the real groups, as we have already seen in the real-rank one case. (c) The situation for the real groups is much more complicated, but there is always a close connection between irreducibility of principal series and existence of complementary series. As a particular result we point out there are reducible representations of the principal series of $S L(n, \mathbf{R})$, whenever $n$ is even. ${ }^{3}$

We use the following notation: $G=K A N$ is an Iwasawa decomposition of a connected semisimple Lie group of matrices, $\theta$ is the Cartan involution of $G$ corresponding to $K, M$ and $M^{\prime}$ are the centralizer and normalizer of $A$ in $K$, and $W=M^{\prime} / M$ is the Weyl group. Let $\sigma$ be an irreducible unitary representation of the compact group $M$, and let $\lambda$ be a unitary character of $A$. Form the representation $\sigma \otimes \lambda \otimes 1$ of the closed subgroup $M A N$. The principal series of unitary representations $U^{\sigma, \lambda}$ of $G$ is parametrized by $(\sigma, \lambda)$ and is obtained by inducing these representations of $M A N$ to $G$.

There is an equivalent way of defining the principal series that allows $\lambda$ to be nonunitary; the resulting representations are not unitary unless $\lambda$ is unitary. Namely, if $V_{\sigma}$ is the space in which $\sigma$ operates, let $H^{\sigma}$ be the set of all squareintegrable functions on $K$ with values in $V_{\sigma}$ such that, for each $m$ in $M, f(m k)=$ $\sigma(m) f(k)$ for almost every $k$ in $K$. These functions extend to $G$ under the definition $^{4}$

$$
f(a n x)=\mu(a)^{1 / 2} \lambda(a) f(x),
$$

and then $U^{\sigma, \lambda}(g) f(x)=f(x g)$. Roughly, $U^{\sigma, \lambda}$ is in the complementary series if the inner product in $H^{\sigma}$ can be redefined on a dense subspace so that $U^{\sigma, \lambda}$ becomes unitary. 
Let $m^{\prime}$ be in $M^{\prime}$ and define $m^{\prime} \sigma(m)=\sigma\left(m^{\prime-1} m m^{\prime}\right)$ and $m^{\prime} \lambda$ similarly. Let $N_{m^{\prime}}=N \cap m^{\prime}(\theta N) m^{\prime-1}$, and, for $f$ in $H^{\sigma}$, consider the formal integral ${ }^{5}$

$$
A\left(m^{\prime}, \sigma, \lambda\right) f(x)=\int_{N_{m}} f\left(m^{\prime-1} u x\right) d u .
$$

Formally we have the identity

$$
A\left(m^{\prime}, \sigma, \lambda\right) U^{\sigma, \lambda}(x)=U^{m^{\prime} \sigma, m^{\prime} \lambda}(x) A\left(m^{\prime}, \sigma, \lambda\right)
$$

for all $x$ in $G$. We call $A\left(m^{\prime}, \sigma, \lambda\right)$ an intertwining operator.

For the moment suppose that $\operatorname{dim} A=1$; this is the real-rank one case. Here $W$ has two elements and we suppose that $m^{\prime}$ is a representative of the nontrivial element. As we showed earlier, ${ }^{2}$ the integral (1) converges for $\lambda$ in a half plane, thereby defining $A\left(m^{\prime}, \sigma, \lambda\right)$ in such a way that (2) holds. $A\left(m^{\prime}, \sigma, \lambda\right)$ extends to a global meromorphic function of $\Lambda=\log \lambda$ as an operator on smooth functions, and (2) holds for the extension. Moreover,

$$
A\left(m^{\prime-1}, m^{\prime} \sigma, m^{\prime} \lambda\right) A\left(m^{\prime}, \sigma, \lambda\right)=c_{\sigma}(\Lambda) I
$$

for a meromorphic complex-valued function $c_{\sigma}(\Lambda)$ that is independent of $m^{\prime}$ and the representative of the equivalence class of $\sigma$.

We shall identify $c_{\sigma}(\Lambda)$. This is a basic step in our work. Fix a normalization of Haar measure on $G$. The representations $U^{\sigma, \lambda}$ that contribute to the Plancherel measure for $G$ are those with $\lambda$ unitary, i.e., $\lambda=\exp$ it $\Lambda_{0}$ for a fixed real-valued $\Lambda_{0}$. The contribution to the Plancherel measure is of the form $p_{\sigma}\left(\right.$ it $\left.\Lambda_{0}\right) \mathrm{dt}$. Here $p_{\sigma}$ extends to a global meromorphic function $p_{\sigma}(\Lambda)$.

THEOREM 1. There is a positive constant $\kappa$ depending on $\sigma$ but independent of $\lambda=\exp \Lambda$, such that $c_{\sigma}(\Lambda)=\kappa p_{\sigma}(\Lambda)^{-1}$.

The Plancherel measure is known explicitly for the groups in question. ${ }^{6}$ The importance of the theorem is that it gives an explicit formula for the function $c_{\sigma}(\Lambda)$, which carries most of the information concerning irreducibility of principal series and existence of complementary series. For instance, the irreducibility criterion is now as follows:

Corollary. If $G$ has real-rank one, then $U^{\sigma, \lambda}$ is reducible if and only if $\lambda=$ $1, \sigma$ is equivalent with $m^{\prime} \sigma$, and $p_{\sigma}(0) \neq 0$.

The technical device for using the functions $c_{\sigma}$ is to construct suitable meromorphic functions $\gamma_{\sigma}(z)$ such that $c_{\sigma}(z)=\gamma_{\sigma}(z) \overline{\gamma_{\sigma}(-\bar{z})}$ and $\gamma_{m^{\prime} \sigma}(z)=\overline{\gamma_{\sigma}(\bar{z})}$. Fix a choice of these functions, and define $\alpha\left(m^{\prime}, \sigma, \lambda\right)=\gamma_{\sigma}(\Lambda)^{-1} A\left(m^{\prime}, \sigma, \lambda\right)$. Then $a\left(m^{\prime}, \sigma, \lambda\right)$ is unitary for unitary $\lambda$, and also (3) gives

$$
Q\left(m^{\prime-1}, m^{\prime} \sigma, m^{\prime} \lambda\right) \propto\left(m^{\prime}, \sigma, \lambda\right)=I .
$$

Now drop the assumption that $\operatorname{dim} A=1$. The main idea in the general case is to write any element of the Weyl group as a product of simple reflections, reducing the questions to the groups of real-rank one. In this connection a significant advance was made by Schiffmann, ${ }^{7}$ who proved that it is possible to normalize simultaneously the Haar measures on all the $N_{m^{\prime}}$ so that the following theorem holds: Let $p=m^{\prime} M$, write $p=\prod_{i=1}^{n} p_{i}$ as the product of simple reflections in as short a fashion as possible (call $n$ the length of $p$ ), and let $m^{\prime}=\Pi m^{\prime}{ }_{i}$ 
be a corresponding decomposition of $m^{\prime}$. If $\langle R e \Lambda, \alpha\rangle>0$ for every positive restricted root $\alpha$, then the integral (1) converges absolutely, each integral $A\left(m_{i}{ }^{\prime}\right.$, $\left.m^{\prime}{ }_{i+1} \ldots m^{\prime}{ }_{n}(\sigma, \lambda)\right)$ converges absolutely, and $A\left(m^{\prime}, \sigma, \lambda\right)$ is the product

$$
A\left(m^{\prime}, \sigma, \lambda\right)=A\left(m_{1}^{\prime}, m^{\prime}{ }_{2} \ldots m^{\prime}{ }_{n}(\sigma, \lambda)\right) \ldots A\left(m^{\prime}{ }_{n}, \sigma, \lambda\right) \text {. }
$$

From this follows the further identity

$$
A\left(m^{\prime}{ }_{1} m^{\prime}{ }_{2}, \sigma, \lambda\right)=A\left(m_{1}^{\prime}, m_{2}^{\prime} \sigma, m_{2}^{\prime} \lambda\right) A\left(m^{\prime}{ }_{2}, \sigma, \lambda\right)
$$

under the assumption that the length of $m_{1}^{\prime} m_{2}^{\prime} M$ is the sum of the lengths of $m_{1}{ }_{1} M$ and $m_{2}{ }_{2} M$.

From the decomposition (5) and the real-rank one case, it follows that $A\left(m^{\prime}\right.$, $\sigma, \lambda)$ extends to a global meromorphic function of $\lambda$. Then (6) holds for the extensions as well. We wish to describe how to normalize the extended operators $A\left(m^{\prime}, \sigma, \lambda\right)$ so that (6) holds with no restriction on $m_{1}^{\prime}$ and $m_{2}^{\prime}$. The role of Theorem 1 is to make this normalization explicit.

First let $m^{\prime}$ in $M^{\prime}$ be such that $m^{\prime} M$ is a simple reflection $p_{\alpha}$. Let $G_{\alpha}$ be the real-rank one group associated to the simple restricted root $\alpha$, and let $M_{\alpha}$ and $A_{\alpha}$ be the $M$ and $A$ of $G_{\alpha}$; we shall suppose $m^{\prime}$ is in $G_{\alpha}$. There is a well-known way ${ }^{8}$ of viewing $U^{\sigma, \lambda}$ as operating on functions on $\theta N$. If we regard $\theta N$ as the product of $m^{\prime-1} N_{m^{\prime}} m^{\prime}$ and the group $\theta N \cap\left(m^{\prime-1} \theta N m^{\prime}\right)$, then it is easy to see that $A\left(m^{\prime}, \sigma, \lambda\right)$ decomposes as

$$
A\left(m^{\prime}, \sigma, \lambda\right)=A\left(m^{\prime},\left.\sigma\right|_{M_{\alpha}},\left.\lambda\right|_{A_{\alpha}}\right) \otimes I .
$$

Now it is known that $M=M_{\alpha} Z_{M}\left(M_{\alpha}\right),{ }^{9}$ and it follows that the restriction $\left.\sigma\right|_{M_{\alpha}}$ is equivalent with a multiple of a single irreducible representation $\bar{\sigma}$ of $M_{\alpha}$. Put $\alpha\left(m^{\prime}, \sigma, \lambda\right)=\gamma_{\bar{\sigma}}\left(\left.\log \lambda\right|_{A_{\alpha}}\right)^{-1} A\left(m^{\prime}, \sigma, \lambda\right)$.

For a general element $m^{\prime}$ in $M^{\prime}$, decompose $A\left(m^{\prime}, \sigma, \lambda\right)$ as in (5), obtain the $\gamma$ factor for each rank-one operator, and use the product of these $\gamma$ factors as a normalizing factor for $A\left(m^{\prime}, \sigma, \lambda\right)$. Call the normalized operator $a\left(m^{\prime}, \sigma, \lambda\right)$. One can show that this normalization is independent of the particular decomposition $m^{\prime}=\Pi m^{\prime}{ }_{i}$. Combining (4), (6), (7), and a supplementary argument, we obtain the following theorem:

THEOREM 2. For any $m_{1}^{\prime}$ and $m_{2}^{\prime}$ in $M^{\prime}$,

$$
Q\left(m^{\prime}{ }_{1} m^{\prime}{ }_{2}, \sigma, \lambda\right)=Q\left(m^{\prime}{ }_{1}, m_{2}^{\prime} \sigma, m_{2}^{\prime} \lambda\right) Q\left(m^{\prime}{ }_{2}, \sigma, \lambda\right) \text {. }
$$

Fix $p$ in $W$ with $p^{2}=1$. Suppose $p=m^{\prime} M$, and let $\sigma$ be an irreducible unitary representation of $M$ such that $m^{\prime} \sigma$ and $\sigma$ are equivalent. Then it is possible to extend $\sigma$ (in exactly two ways, differing by a sign) to a representation (on the same vector space) of the group generated by $M$ and $m^{\prime}$. Thus form the operator

$$
\sigma\left(m^{\prime}\right) Q\left(m^{\prime}, \sigma, 1\right) .
$$

This operator is hermitian and unitary. The critical question is whether it is scalar. 
If (8) is scalar, adjust $\sigma\left(m^{\prime}\right)$ so that the scalar is one. For those $\lambda$ such that $p \lambda=\bar{\lambda}^{-1}$, the operator

$$
\sigma\left(m^{\prime}\right) \mathfrak{Q}\left(m^{\prime}, \sigma, \lambda\right)
$$

is hermitian, and it is positive definite for $\lambda=1$. Thus (9) remains positive definite on any connected set of such $\lambda$ containing 1 on which (9) is nonsingular on every $K$-finite subspace of functions. Writing $p$ as a product of simple reflections and decomposing (9) accordingly, we see that the nonsingularity of (9) reduces to the real-rank one case. Explicit information about the nonsingularity of (9) is therefore available from Theorem 1 and from our earlier results. ${ }^{2}$ In any case the operator is nonsingular at least for $\lambda$ in a small neighborhood of 1 . Consequently (9) is positive definite if $\lambda$ is sufficiently close to 1 and if $p \lambda=$ $\bar{\lambda}^{-1}$. For such $\lambda$ it follows from standard arguments that $U^{\sigma, \lambda}$ is in the complementary series. (On the other hand if (8) is not scalar, then it follows from (2) that $U^{\sigma, 1}$ is reducible.)

Thus we get either existence of complementary series or reducibility of a member of the principal series, depending on whether (8) is scalar or not. In order to decide whether this operator is scalar, one makes systematic use of the following two facts: (a) In a factorization of $Q\left(m^{\prime}, \sigma, 1\right)$ according to a minimal decomposition $p=\Pi p_{\alpha}$ with the $p_{\alpha}$ simple reflections, if each factor is nonconstant, then $Q\left(m^{\prime}, \sigma, 1\right)$ is nonconstant. (b) Every element $p$ of $W$ with $p^{2}$ $=1$ is the product of commuting reflections in hyperplanes.

Examples: (1) $S L(n, \mathbf{R}), n=2 k$. This is the group of real nonsingular matrices of determinant one. $M$ can be taken as the diagonal matrices with $\epsilon_{i}= \pm 1$. Define $\sigma\left(\epsilon_{1}, \ldots, \epsilon_{n}\right)=\epsilon_{1} \epsilon_{3} \epsilon_{5} \ldots \epsilon_{n-1}$ and a permutation $p=\left(\begin{array}{ll}1 & 2\end{array}\right)$ (3 4) (5 6)...(n-1,n). Then $p^{2}=1$ and $p \sigma=\sigma$ since $\Pi \epsilon_{i}=1$. Applying Theorem 2, we can see that $Q\left(m^{\prime}, \sigma, \lambda\right)$ is not constant if $p \lambda=\lambda$. Thus $U^{\sigma, \lambda}$ is reducible, for these $\sigma$ and $\lambda .^{3}$

(2) Complex semisimple groups. Suppose $p$ is in $W, p^{2}=1$, and $p \sigma=\sigma$. Decompose $p$, by the fact (b), as the product of distinct commuting (not necessarily simple) reflections $p_{\alpha}$. For such a group, one can show that $p_{\alpha} \sigma=\sigma$ for each $p_{\alpha}$. When $p_{\alpha} \sigma=\sigma$, the fact that $Q\left(p_{\alpha}, \sigma, 1\right)$ is scalar follows from Theorem 2 and from the theory for $S L(2, \mathrm{C})$. Thus by a second application of Theorem $2, a(p, \sigma, 1)$ is scalar; together with an additional argument, the above discussion leads us to the following conclusion.

Theorem $3 .^{10}$ Let $G$ be a complex semisimple group, $p$ a member of the Weyl group such that $p^{2}=1, \sigma$ a character of $M$, and $\lambda=\exp \Lambda$ a character of $A$. Suppose that the following necessary conditions for a representation to be in the complementary series are satisfied: $p \sigma=\sigma, p \Lambda=-\bar{\Lambda}$. If

$$
\frac{|\langle\operatorname{Re\Lambda }, \alpha\rangle|}{\langle\alpha, \alpha\rangle}<1
$$

for every root $\alpha$, then $U^{\sigma, \lambda}$ is in the complementary series.

* Supported by National Science Foundation grant GP 11767.

t Supported by Air Force Office of Scientific Research grant AF 68-1467. 
1 Knapp, A. W., and E. M. Stein, these Proceedings, 63, 281-284 (1969).

2 Knapp, A. W., and E. M. Stein, "Existence of complementary series," in Problems in Analysis: Symposium in Honor of Salomon Bochner, to appear. The definition of intertwining operator in that note differs from the present one by a factor of $\sigma\left(m^{\prime}\right)$.

${ }^{3}$ Cf. Gelfand, I. M., and M. I. Graev, Translations Amer. Math. Soc. 2, 147-205 (1956), where the contrary is suggested. N. Wallach raised to us the question of irreducibility of the representations of $S L(n, \mathbf{R})$ that we consider.

"See Kunze, R. A., and E. M. Stein, Amer. J. Math., 89, 385-442 (1967), particularly p. 388, for the definition of $\mu$.

' Ibid., 390 (1967).

' Okamoto, K., Osaka J. Math., 2, 247-282 (1965); Harish-Chandra, Ann. Math., 83, 74-128(1966); Hirai, T., Proc. Japan Acad., 42, 323-326 (1966).

${ }^{7}$ Schiffmann, G., Compt. Rend., 266, 47-49, 859-861 (1968); details are in his as yet unpublished thesis.

${ }^{8}$ Kunze, R. A., and E. M. Stein, Amer. J. Math., 89, 401 (1967).

-We are indebted to H. Garland for bringing this fact to our attention.

${ }^{10}$ For some earlier results see Kunze, R., "Analytic continuation of intertwining operators," preprint (1968); Kostant, B., Bull. Amer. Math. Soc., 75, 627-642 (1969); and Helgason, S., these Procendings, 63, 643-647 (1969). 